This is an electronic reprint of the original article. This reprint may differ from the original in pagination and typographic detail.

Author(s): Ferreira, David Dos Santos; Kenig, Carlos E.; Salo, Mikko

Title: Determining an unbounded potential from Cauchy data in admissible geometries

Year: $\quad 2013$

Version:

Please cite the original version:

Ferreira, D. D. S., Kenig, C. E., \& Salo, M. (2013). Determining an unbounded potential from Cauchy data in admissible geometries. Communications in Partial Differential Equations, 38(1), 50-68. https://doi.org/10.1080/03605302.2012.736911

All material supplied via JYX is protected by copyright and other intellectual property rights, and duplication or sale of all or part of any of the repository collections is not permitted, except that material may be duplicated by you for your research use or educational purposes in electronic or print form. You must obtain permission for any other use. Electronic or print copies may not be offered, whether for sale or otherwise to anyone who is not an authorised user. 


\title{
DETERMINING AN UNBOUNDED POTENTIAL FROM CAUCHY DATA IN ADMISSIBLE GEOMETRIES
}

\author{
DAVID DOS SANTOS FERREIRA, CARLOS E. KENIG, AND MIKKO SALO
}

\begin{abstract}
In [4] anisotropic inverse problems were considered in certain admissible geometries, that is, on compact Riemannian manifolds with boundary which are conformally embedded in a product of the Euclidean line and a simple manifold. In particular, it was proved that a bounded smooth potential in a Schrödinger equation was uniquely determined by the Dirichlet-to-Neumann map in dimensions $n \geq 3$. In this article we extend this result to the case of unbounded potentials, namely those in $L^{n / 2}$. In the process, we derive $L^{p}$ Carleman estimates with limiting Carleman weights similar to the Euclidean estimates of Jerison-Kenig [11] and Kenig-Ruiz-Sogge [13].
\end{abstract}

\section{Contents}

1. Introduction

2. $L^{p}$ Carleman estimates

3. Complex geometrical optics 11

4. Recovering the potential $\quad 15$

5. Attenuated ray transform 16

$\begin{array}{ll}\text { References } & 20\end{array}$

\section{INTRODUCTION}

In this paper we consider the problem of proving $L^{p}$ estimates for limiting Carleman weights on Riemannian manifolds, and the related inverse problem of recovering an $L^{n / 2}$ potential from the Dirichlet-toNeumann map (DN map) related to the Schrödinger equation. The main motivation comes from the inverse conductivity problem posed by Calderón [1]. This problem asks to determine the conductivity function of a medium from electrical measurements made on its boundary. 
In mathematical terms, if $\Omega \Subset \mathbf{R}^{n}$ is the medium of interest having a positive conductivity coefficient $\gamma$, in the Calderón problem one considers the conductivity equation

$$
\nabla \cdot \gamma \nabla u=0 \quad \text { in } \Omega
$$

and defines the DN map by

$$
\Lambda_{\gamma}:\left.\left.u\right|_{\partial \Omega} \mapsto \gamma \frac{\partial u}{\partial \nu}\right|_{\partial \Omega} .
$$

This operator maps the voltage at the boundary to the current given by $\gamma$ times the normal derivative, which encodes the electrical measurements at the boundary. The inverse problem of Calderón asks to determine $\gamma$ from the knowledge of $\Lambda_{\gamma}$. This problem has been extensively studied and we refer to [31] for a recent survey.

The anisotropic Calderón problem considers the case where the conductivity $\gamma$ is a symmetric positive definite matrix instead of a scalar function. This corresponds to situations where the electrical properties of the medium depend on direction. The problem is open in general in dimensions $n \geq 3$, see [4] for known results and more details. Following [19] the problem may be recast as the determination of the metric $g$ on a compact Riemannian manifold $(M, g)$ with boundary from the corresponding DN map. In [4] progress was made on the anisotropic Calderón problem in the following class of conformal smooth manifolds.

Definition. A compact Riemannian manifold $(M, g)$, with dimension $n \geq 3$ and with boundary $\partial M$, is called admissible if $M \Subset \mathbf{R} \times M_{0}$ for some $(n-1)$-dimensional simple manifold $\left(M_{0}, g_{0}\right)$, and if $g=c\left(e \oplus g_{0}\right)$ where $e$ is the Euclidean metric on $\mathbf{R}$ and $c$ is a smooth positive function on $M$.

Here, a compact manifold $\left(M_{0}, g_{0}\right)$ with boundary is simple if for any $p \in M_{0}$ the exponential map $\exp _{p}$ with its maximal domain of definition is a diffeomorphism onto $M_{0}$, and if $\partial M_{0}$ is strictly convex (that is, the second fundamental form of $\partial M_{0} \hookrightarrow M_{0}$ is positive definite).

In [4] it was proved that a Riemannian metric in a conformal class of admissible geometries is uniquely determined by the DN map. This was obtained as a corollary of a result for the Schrödinger equation in a fixed admissible manifold, stating that a bounded smooth potential $q$ is determined by the corresponding DN map. In [4] all coefficients were assumed infinitely differentiable. In this paper we relax this requirement and show that a complex potential $q \in L^{n / 2}(M)$ is determined by the DN map. 
To state the main result, assume that $(M, g)$ is a compact Riemannian manifold with smooth boundary $\partial M$, and let $\Delta_{g}$ be the LaplaceBeltrami operator. Given a complex function $q \in L^{n / 2}(M)$, where $n \geq 3$ is the dimension of the manifold $M$, we consider the Dirichlet problem

$$
\left(-\Delta_{g}+q\right) u=0 \text { in } M,\left.\quad u\right|_{\partial M}=f .
$$

We assume throughout that 0 is not a Dirichlet eigenvalue for this problem, and then standard arguments (see for instance [8]) show that there is a well-defined DN map

$$
\Lambda_{g, q}: H^{1 / 2}(\partial M) \rightarrow H^{-1 / 2}(\partial M),\left.\quad f \mapsto \partial_{\nu} u\right|_{\partial M} .
$$

The following uniqueness theorem is the main result for the inverse problem. (The assumption $q \in L^{n / 2}$ may be considered optimal in the context of the standard wellposedness theory for the Dirichlet problem with $L^{p}$ potentials, and also for the strong unique continuation principle to hold [11].)

Theorem 1.1. Let $(M, g)$ be admissible and let $q_{1}, q_{2}$ be complex functions in $L^{n / 2}(M)$. If $\Lambda_{g, q_{1}}=\Lambda_{g, q_{2}}$, then $q_{1}=q_{2}$.

Remark 1.2. The proof of Theorem 1.1 reduces to the case where $g=e \oplus g$ is a product metric. Indeed, from the well known relation

$$
\Lambda_{g, q}=\Lambda_{c^{-1} g, c\left(q-q_{c}\right)} \text { with } q_{c}=c^{\frac{n-2}{4}} \Delta_{g}\left(c^{-\frac{n-2}{4}}\right),
$$

one deduces that if $\Lambda_{g, q_{1}}=\Lambda_{g, q_{2}}$ then $\Lambda_{c^{-1} g, c\left(q_{1}-q_{c}\right)}=\Lambda_{c^{-1} g, c\left(q_{2}-q_{c}\right)}$. Since this simplifies the context, we will deal with product type metric $g=e \oplus g$ in what follows.

In the case where $M$ is a bounded domain in $\mathbf{R}^{n}$ and $g$ is the Euclidean metric, this result is due to Lavine and Nachman [18] following the earlier result of Jerison and Kenig for $q_{j} \in L^{n / 2+\varepsilon}(M)$ for some $\varepsilon>0$ (see Chanillo [2] for an account and also for a related result with $q_{j}$ in a Fefferman-Phong class with small norm). As mentioned above, if $q$ is a smooth function on an admissible manifold $M$ this result was proved in [4] by using $L^{2}$ Carleman estimates. In fact, smoothness of $q$ is not essential, and by inspecting the proof of [4] the uniqueness result can be extended to bounded continuous $q$ (with the complex geometrical optics construction in the proof going through for $\left.q \in L^{n}(M)\right)$. However, the proof for $q \in L^{n / 2}$ requires to replace the $L^{2}$ Carleman estimates in [4] with corresponding $L^{p}$ Carleman estimates.

The other main result in this paper is a $L^{p}$ Carleman estimate for limiting Carleman weights on Riemannian manifolds. The concept of limiting Carleman weights was introduced in [15] as part of a general 
procedure for producing special complex geometrical optics solutions to elliptic equations, with applications to inverse problems. We refer to [4] for a precise definition and more careful analysis of limiting Carleman weights, also on Riemannian manifolds. For present purposes it is sufficient to mention that the existence of a limiting Carleman weight on $(M, g)$ in dimensions $n \geq 3$ is locally equivalent with the manifold being admissible, and that typical limiting Carleman weights in $\mathbf{R}^{n}$, $n \geq 3$, include the linear weight $\varphi(x)=x_{1}$ and logarithmic weight $\varphi(x)=\log |x|$.

The last two weights are featured in the literature of Carleman estimates and unique continuation, in particular in the scale invariant $L^{p}$ Carleman estimates of Kenig-Ruiz-Sogge [13] for the linear weight and of Jerison-Kenig [11] for the logarithmic weight. We prove an analogue of these estimates on more general Riemannian manifolds. Note that the existence of a limiting Carleman weight requires at least locally a product structure on the manifold, and therefore the following result is stated for the linear weight on a product manifold. The result, in the case when the manifold $\left(M_{0}, g_{0}\right)$ below is the standard $n-1$ dimensional torus, is due to Shen [24].

Theorem 1.3. Let $\left(M_{0}, g_{0}\right)$ be an $(n-1)$-dimensional compact manifold without boundary, and equip $\mathbf{R} \times M_{0}$ with the metric $g=e \oplus g_{0}$ where $e$ is the Euclidean metric. The Euclidean coordinate is denoted by $x_{1}$. For any compact interval $I \subseteq \mathbf{R}$ there exists a constant $C_{I}>0$ such that if $|\tau| \geq 4$ and

$$
\tau^{2} \notin \operatorname{Spec}\left(-\Delta_{g_{0}}\right)
$$

then we have

$$
\left\|\mathrm{e}^{\tau x_{1}} u\right\|_{L^{\frac{2 n}{n-2}}\left(\mathbf{R} \times M_{0}\right)} \leq C_{I}\left\|\mathrm{e}^{\tau x_{1}} \Delta_{g} u\right\|_{L^{\frac{2 n}{n+2}}\left(\mathbf{R} \times M_{0}\right)}
$$

when $u \in C_{0}^{\infty}\left(I \times M_{0}\right)$.

The proof of the $L^{2}$ Carleman estimates for limiting Carleman weights in [4] is based on integration by parts and cannot be used in the $L^{p}$ setting. However, in [14] another proof of the $L^{2}$ Carleman estimate is given; this proof is based on Fourier analysis and gives an explicit inverse for the conjugated Laplacian. We will derive the $L^{p}$ bounds from this explicit inverse operator. This follows the proof of the $L^{p}$ Carleman estimate of Jerison-Kenig [11] using Jerison's approach [10], [27, Section 5.1] based on the spectral cluster estimates of Sogge [27]. Finally, if one allows strongly pseudoconvex Carleman weights then much stronger estimates are available (see for instance $[16,17]$ ), however for 
the applications to inverse problems it seems necessary to restrict to limiting Carleman weights.

Remark 1.4. The above theorems are in the setting of (conformal) product manifolds. However, the results also apply to warped products. If $f: \mathbf{R} \rightarrow \mathbf{R}$ is a smooth function and $\left(M_{0}, g_{0}\right)$ is an $(n-1)$-dimensional manifold, the warped product $\mathbf{R} \times{ }_{\mathrm{e}^{2 f}} M_{0}$ is the manifold $M=\mathbf{R} \times M_{0}$ endowed with the metric

$$
g\left(x_{1}, x^{\prime}\right)=\left(\begin{array}{cc}
1 & 0 \\
0 & \mathrm{e}^{2 f\left(x_{1}\right)} g_{0}\left(x^{\prime}\right)
\end{array}\right) .
$$

We choose coordinates $y_{1}=\eta\left(x_{1}\right), y^{\prime}=x^{\prime}$ for a suitable smooth strictly increasing function $\eta$. In fact, if

$$
\eta(t)=\int_{0}^{t} \mathrm{e}^{-f(s)} \mathrm{d} s
$$

then $\eta^{\prime}(t)^{-2}=\mathrm{e}^{2 f(t)}$ and the metric in $y$ coordinates becomes a conformal multiple of a product metric,

$$
g\left(y_{1}, y^{\prime}\right)=\mathrm{e}^{2 f\left(\eta^{-1}\left(y_{1}\right)\right)}\left(\begin{array}{cc}
1 & 0 \\
0 & g_{0}\left(y^{\prime}\right)
\end{array}\right) .
$$

Warped products have a natural limiting Carleman weight $\varphi(y)=y_{1}$, and Theorem 1.1 remains true in conformal multiples of warped products whenever $\left(M_{0}, g_{0}\right)$ is a simple manifold.

The paper is organized as follows. Section 1 is the introduction. In Section 2 we prove the $L^{p}$ Carleman estimate complemented with the usual $L^{2}$ Carleman estimates. Section 3 presents the construction of complex geometrical optics solutions for Schrödinger equations with $L^{n / 2}$ potentials in admissible geometries. The proof of Theorem 1.1 is contained in Section 4, modulo a uniqueness result for an analogue of the attenuated geodesic ray transform acting on unbounded functions. This last result has a different character than the rest of the proof, and it is therefore established separately in Section 5. There are two appendices concerning the wellposedness of the Dirichlet problem and the normal operator for the attenuated ray transform.

Acknowledgements. The last named author would like to thank Adrian Nachman for explaining his unpublished argument with Richard Lavine [18] which proves a uniqueness result for $L^{n / 2}$ potentials in Euclidean space. C.K. is supported partly by NSF grant DMS-0968472, and M.S. is supported in part by the Academy of Finland. D. DSF. would like to thank the University of Chicago for its hospitality. 


\section{2. $L^{p}$ Carleman estimates}

The aim of this section is to prove Theorem 1.3, which is an analogue of the $L^{p}$ Carleman estimates obtained in the Euclidean case by Jerison and Kenig [11] (for logarithmic weights) or by Kenig, Ruiz and Sogge [13] (for linear weights). In fact, we prove a more general result which implies Theorem 1.3 by taking $f=\mathrm{e}^{\tau x_{1}} \Delta_{g} \mathrm{e}^{-\tau x_{1}} u$ for $u \in C_{0}^{\infty}\left(I \times M_{0}\right)$. The case when $\left(M_{0}, g_{0}\right)$ is the standard $n-1$ dimensional torus is due to Shen [24].

Proposition 2.1. Let $I \subseteq \mathbf{R}$ be a compact interval and $\left(M_{0}, g_{0}\right)$ a compact $(n-1)$-dimensional manifold without boundary. Equip $N=$ $I \times M_{0}$ with the product metric $g=e \oplus g_{0}$. For $|\tau| \geq 4$ with $\tau^{2} \notin$ $\operatorname{Spec}\left(-\Delta_{g_{0}}\right)$, there is a linear operator $G_{\tau}: L^{2}(N) \rightarrow H^{2}(N)$ such that

$$
\begin{array}{ll}
\mathrm{e}^{\tau x_{1}}\left(-\Delta_{g}\right) \mathrm{e}^{-\tau x_{1}} G_{\tau} v=v & \text { for } v \in L^{2}(N), \\
G_{\tau} \mathrm{e}^{\tau x_{1}}\left(-\Delta_{g}\right) \mathrm{e}^{-\tau x_{1}} v=v & \text { for } v \in C_{0}^{\infty}\left(N^{\text {int }}\right) .
\end{array}
$$

This operator satisfies

$$
\begin{aligned}
\left\|G_{\tau} f\right\|_{L^{2}(N)} & \leq \frac{C_{0}}{|\tau|}\|f\|_{L^{2}(N)}, \\
\left\|G_{\tau} f\right\|_{H^{1}(N)} & \leq C_{0}\|f\|_{L^{2}(N)}, \\
\left\|G_{\tau} f\right\|_{L^{\frac{2 n}{n-2}(N)}} & \leq C_{0}\|f\|_{L^{\frac{2 n}{n+2}}(N)}
\end{aligned}
$$

where $C_{0}$ is independent of $\tau$ (but may depend on $I$ ).

Remark 2.2. In the Euclidean case, $L^{p}$ Carleman estimates with linear weights can be obtained from $L^{p}$ Carleman estimates with pseudoconvex Carleman weights by scaling. Indeed, suppose that the following Carleman estimate

$$
\left\|\mathrm{e}^{\tau\left(x_{1}+x_{1}^{2} / 2 \varepsilon\right)} u\right\|_{L^{\frac{2 n}{n-2}\left(\mathbf{R}^{n}\right)}} \leq C_{K}\left\|\mathrm{e}^{\tau\left(x_{1}+x_{1}^{2} / 2 \varepsilon\right)} \Delta u\right\|_{L^{\frac{2 n}{n+2}\left(\mathbf{R}^{n}\right)}},
$$

holds for all $\varepsilon \leq \varepsilon_{0}$ and all $u \in C_{0}^{\infty}(K)$, then applying this estimate to $u_{\mu}=u(\mu \cdot)$ with $\mu \geq 1$ and $u \in C_{0}^{\infty}(K)$, one gets

$$
\left\|\mathrm{e}^{\frac{\tau}{\mu} x_{1}+\frac{\tau}{\mu^{2}} x_{1}^{2} / 2 \varepsilon} u\right\|_{L^{\frac{2 n}{n-2}}\left(\mathbf{R}^{n}\right)} \leq C_{K}\left\|\mathrm{e}^{\frac{\tau}{\mu} x_{1}+\frac{\tau}{\mu^{2}} x_{1}^{2} / 2 \varepsilon} \Delta u\right\|_{L^{\frac{2 n}{n+2}}\left(\mathbf{R}^{n}\right)} .
$$

Choosing $\mu=\sqrt{\tau}$, and using the fact that $\mathrm{e}^{x_{1}^{2} / 2 \varepsilon} \simeq C_{\varepsilon}$ on $K$, one gets the Carleman estimate

$$
\left\|\mathrm{e}^{\mu x_{1}} u\right\|_{L^{\frac{2 n}{n-2}}\left(\mathbf{R}^{n}\right)} \leq C_{K, \varepsilon}\left\|\mathrm{e}^{\mu x_{1}} \Delta u\right\|_{L^{\frac{2 n}{n+2}}\left(\mathbf{R}^{n}\right)},
$$

for all $u \in C_{0}^{\infty}(K)$. However, in the anisotropic case, one has to find another way. 
To prepare for the proof of Proposition 2.1 consider the LaplaceBeltrami operator on $N$,

$$
P=\Delta_{g}=\partial_{x_{1}}^{2}+\Delta_{g_{0}}
$$

and the corresponding conjugated operator (by the limiting Carleman weight $x_{1}$ )

$$
\mathrm{e}^{\tau x_{1}} P \mathrm{e}^{-\tau x_{1}}=\partial_{x_{1}}^{2}-2 \tau \partial_{x_{1}}+\tau^{2}+\Delta_{g_{0}} .
$$

We denote by $\lambda_{0}=0<\lambda_{1} \leq \lambda_{2} \leq \ldots$ the sequence of eigenvalues of $-\Delta_{g_{0}}$ on $M_{0}$ and $\left(\psi_{j}\right)_{j \geq 0}$ the corresponding sequence of eigenfunctions forming an orthonormal basis of $L^{2}\left(M_{0}\right)$,

$$
-\Delta_{g_{0}} \psi_{j}=\lambda_{j} \psi_{j}
$$

We denote by $\pi_{j}: L^{2}\left(M_{0}\right) \rightarrow L^{2}\left(M_{0}\right), u \mapsto\left(u, \psi_{j}\right) \psi_{j}$ the projection on the linear space spanned by the eigenfunction $\psi_{j}$ so that

$$
\sum_{j=0}^{\infty} \pi_{j}=\mathrm{Id}, \quad \sum_{j=0}^{\infty} \lambda_{j} \pi_{j}=-\Delta_{g_{0}}
$$

and by

$$
\widehat{u}(j)=\int_{M_{0}} u \overline{\psi_{j}} \mathrm{~d} V_{g_{0}}
$$

the corresponding Fourier coefficients of a function $u$ on $M_{0}$. We define the spectral clusters as

$$
\chi_{k}=\sum_{k \leq \sqrt{\lambda_{j}}<k+1} \pi_{j}, \quad k \in \mathbf{N} .
$$

Note that these are projection operators, $\chi_{k}^{2}=\chi_{k}$, and they constitute a decomposition of the identity

$$
\mathrm{Id}=\sum_{k=0}^{\infty} \chi_{k} .
$$

We end this paragraph by recalling the spectral cluster estimates of Sogge $[25,27]$ that we will need:

$$
\begin{aligned}
\left\|\chi_{k} u\right\|_{L^{\frac{2 n}{n-2}}\left(M_{0}\right)} & \leq C(1+k)^{\frac{1}{2}-\frac{1}{n}}\|u\|_{L^{2}\left(M_{0}\right)}, \\
\left\|\chi_{k} u\right\|_{L^{2}\left(M_{0}\right)} & \leq C(1+k)^{\frac{1}{2}-\frac{1}{n}}\|u\|_{L^{\frac{2 n}{n+2}\left(M_{0}\right)}} .
\end{aligned}
$$

The first estimate is given in [27, Corollary 5.1.2] and the second one is a consequence of the first one by duality. 
Proof of Proposition 2.1. Recall that our main goal is to prove

$$
\|u\|_{L^{\frac{2 n}{n-2}\left(\mathbf{R} \times M_{0}\right)}} \leq C_{I}\|f\|_{L^{\frac{2 n}{n+2}\left(\mathbf{R} \times M_{0}\right)}}
$$

when $u \in C_{0}^{\infty}\left(I \times M_{0}\right)$ and

$$
D_{x_{1}}^{2} u+2 i \tau D_{x_{1}} u-\Delta_{g_{0}} u-\tau^{2} u=f
$$

with $D_{x_{1}}=-i \partial_{x_{1}}$. The inverse operator in (2.6) is actually easy to write down, as was done in [14]. The same procedure appears in [10] and [27, Section 5.1]. Writing $f=\sum_{j=0}^{\infty} \pi_{j} f$ and similarly for $u$, the equation formally becomes

$$
\left(D_{x_{1}}^{2}+2 i \tau D_{x_{1}}-\tau^{2}+\lambda_{j}\right) \pi_{j} u=\pi_{j} f
$$

for $x_{1}$ on the real line and for $j \geq 0$. The symbol of the operator on the left is $\xi_{1}^{2}+2 i \tau \xi_{1}-\tau^{2}+\lambda_{j}$, and this is always nonzero provided that $\tau^{2} \neq \lambda_{j}$ for all $j$. Thus, if

$$
\tau^{2} \notin \operatorname{Spec}\left(-\Delta_{g_{0}}\right),
$$

an inverse operator may be obtained as

$$
\tilde{G}_{\tau} f\left(x_{1}, x^{\prime}\right)=\sum_{j=0}^{\infty} \int_{-\infty}^{\infty} m_{\tau}\left(x_{1}-y_{1}, \sqrt{\lambda_{j}}\right) \pi_{j} f\left(y_{1}, x^{\prime}\right) \mathrm{d} y_{1}
$$

where

$$
m_{\tau}(t, \mu)=\frac{1}{2 \pi} \int_{-\infty}^{\infty} \frac{\mathrm{e}^{i t \eta}}{\eta^{2}+2 i \tau \eta-\tau^{2}+\mu^{2}} \mathrm{~d} \eta, \quad \mu>0 .
$$

The operator $\tilde{G}_{\tau}$ is the same as $G_{\tau}$ in [14, Section 4], except that in the present setting $\left\{\psi_{j}\right\}$ is a basis of $L^{2}\left(M_{0}\right)$ on a compact manifold $\left(M_{0}, g_{0}\right)$ without boundary instead of being a basis of Dirichlet eigenfunctions on a compact manifold with boundary. Let

$$
L_{\delta}^{2}\left(\mathbf{R} \times M_{0}\right)=\left\{f \in L_{\mathrm{loc}}^{2}\left(\mathbf{R} \times M_{0}\right) ;\left(1+x_{1}^{2}\right)^{\delta / 2} f \in L^{2}\left(\mathbf{R} \times M_{0}\right)\right\}
$$

and let $H_{\delta}^{s}\left(\mathbf{R} \times M_{0}\right)$ by the corresponding Sobolev spaces. The proof of [14, Proposition 4.1] goes through for $\tilde{G}_{\tau}$ without changes and shows that for any fixed $\delta>1 / 2$, if $|\tau| \geq 1$ and $\tau^{2} \notin \operatorname{Spec}\left(-\Delta_{g_{0}}\right)$ then the equation

$$
\mathrm{e}^{\tau x_{1}}\left(-\Delta_{g}\right) \mathrm{e}^{-\tau x_{1}} v=f
$$

has a unique solution $v=\tilde{G}_{\tau} f \in H_{-\delta}^{1}\left(\mathbf{R} \times M_{0}\right)$ for any $f \in L_{\delta}^{2}\left(\mathbf{R} \times M_{0}\right)$. Further, $v \in H_{-\delta}^{2}\left(\mathbf{R} \times M_{0}\right)$ and

$$
\|v\|_{H_{-\delta}^{s}\left(\mathbf{R} \times M_{0}\right)} \leq C_{0}|\tau|^{s-1}\|f\|_{L_{\delta}^{2}\left(\mathbf{R} \times M_{0}\right)}, \quad 0 \leq s \leq 2 .
$$

We define

$$
G_{\tau} f\left(x_{1}, x^{\prime}\right)=\chi\left(x_{1}\right) \tilde{G}_{\tau} f\left(x_{1}, x^{\prime}\right)
$$


with $\chi \in C_{0}^{\infty}(\mathbf{R})$ which equals 1 on $I$. It is then clear that all the statements in the proposition except for the $L^{p}$ estimate follow from the results for $\tilde{G}_{\tau}$ explained above.

It is sufficient to prove the $L^{p}$ estimate in the case where $\tau \geq 4$ and $\tau^{2} \notin \operatorname{Spec}\left(-\Delta_{g_{0}}\right)$. We first record a lemma.

Lemma 2.3. If $\tau>0, \mu>0, \tau \neq \mu$ and $t \in \mathbf{R}$ then

$$
\left|m_{\tau}(t, \mu)\right| \leq \frac{1}{\mu} \mathrm{e}^{-|\tau-\mu||t|} .
$$

Besides, if $\tau>0$ then

$$
\left|m_{\tau}(t, 0)\right| \leq|t| \mathrm{e}^{-\tau|t|}
$$

Proof. This follows by writing

$$
\frac{1}{(i \eta-(\tau+\mu))(i \eta-(\tau-\mu))}=\frac{1}{2 \mu}\left[\frac{1}{i \eta-(\tau+\mu)}-\frac{1}{i \eta-(\tau-\mu)}\right]
$$

and by noting that for $\alpha>0$

$$
\mathscr{F}_{\eta}^{-1}\left\{\frac{1}{i \eta+\alpha}\right\}(t)= \begin{cases}0, & t<0 \\ \mathrm{e}^{-\alpha t}, & t>0\end{cases}
$$

and similarly for $\alpha<0$.

Furthermore we have

$$
\mathscr{F}_{\eta}^{-1}\left\{\frac{1}{(\eta+i \tau)^{2}}\right\}(t)= \begin{cases}t \mathrm{e}^{-\tau|t|}, & t<0 \\ 0, & t>0\end{cases}
$$

and this concludes the proof of the lemma.

From the decomposition (2.3), the spectral cluster estimate (2.4), and the fact that spectral clusters are projections $\left(\chi_{k}^{2}=\chi_{k}\right)$, we get the following string of estimates

$$
\begin{aligned}
\|u\|_{L^{\frac{2 n}{n-2}}\left(M_{0}\right)} & =\left\|\sum_{k=0}^{\infty} \chi_{k}^{2} u\right\|_{L^{\frac{2 n}{n-2}}\left(M_{0}\right)} \\
& \lesssim \sum_{k=0}^{\infty}(1+k)^{\frac{1}{2}-\frac{1}{n}}\left\|\chi_{k} u\right\|_{L^{2}\left(M_{0}\right)} .
\end{aligned}
$$

Since

$$
\left\|\chi_{k} u\right\|_{L^{2}\left(M_{0}\right)}=\left(\sum_{k \leq \sqrt{\lambda_{j}}<k+1}|\widehat{u}(j)|^{2}\right)^{\frac{1}{2}}
$$


if we apply the inequality $(2.7)$ to $u=G_{\tau} f\left(x_{1}, \cdot\right)$, we get for almost every $x_{1} \in I$

$$
\begin{aligned}
\left\|G_{\tau} f\left(x_{1}, \cdot\right)\right\|_{L^{\frac{2 n}{n-2}}\left(M_{0}\right)} \lesssim \sum_{k=0}^{\infty}(1+k)^{\frac{1}{2}-\frac{1}{n}} \\
\quad \times\left(\sum_{k \leq \sqrt{\lambda_{j}}<k+1}\left|\int_{-\infty}^{\infty} m_{\tau}\left(x_{1}-y_{1}, \sqrt{\lambda_{j}}\right) \widehat{f}\left(y_{1}, j\right) \mathrm{d} y_{1}\right|^{2}\right)^{\frac{1}{2}} .
\end{aligned}
$$

By Minkowski's inequality, we have

$$
\begin{aligned}
\left\|G_{\tau} f\left(x_{1}, \cdot\right)\right\|_{L^{\frac{2 n}{n-2}}\left(M_{0}\right)} \lesssim \sum_{k=0}^{\infty}(1+k)^{\frac{1}{2}-\frac{1}{n}} \\
\quad \times \int_{-\infty}^{\infty}\left(\sum_{k \leq \sqrt{\lambda_{j}}<k+1}\left|m_{\tau}\left(x_{1}-y_{1}, \sqrt{\lambda_{j}}\right) \widehat{f}\left(y_{1}, j\right)\right|^{2}\right)^{\frac{1}{2}} \mathrm{~d} y_{1}
\end{aligned}
$$

and since

$$
\begin{aligned}
& \sum_{k \leq \sqrt{\lambda_{j}}<k+1}\left|m_{\tau}\left(x_{1}-y_{1}, \sqrt{\lambda_{j}}\right) \widehat{f}\left(y_{1}, j\right)\right|^{2} \\
& \quad \leq \sup _{k \leq \sqrt{\lambda_{j}}<k+1}\left|m_{\tau}\left(x_{1}-y_{1}, \sqrt{\lambda_{j}}\right)\right|^{2} \sum_{k \leq \sqrt{\lambda_{j}}<k+1}\left|\widehat{f}\left(y_{1}, j\right)\right|^{2} \\
& \quad \leq \sup _{k \leq \sqrt{\lambda_{j}}<k+1}\left|m_{\tau}\left(x_{1}-y_{1}, \sqrt{\lambda_{j}}\right)\right|^{2} \times\left\|\chi_{k} f\left(y_{1}, \cdot\right)\right\|_{L^{2}\left(M_{0}\right)}^{2}
\end{aligned}
$$

using once again the spectral cluster estimate (2.4), we finally get

$$
\begin{aligned}
& \left\|G_{\tau} f\left(x_{1}, \cdot\right)\right\|_{L^{\frac{2 n}{n-2}}\left(M_{0}\right)} \lesssim \sum_{k=0}^{\infty}(1+k)^{1-\frac{2}{n}} \\
& \times \int_{-\infty}^{\infty} \sup _{k \leq \sqrt{\lambda_{j}}<k+1}\left|m_{\tau}\left(x_{1}-y_{1}, \sqrt{\lambda_{j}}\right)\right| \times\left\|f\left(y_{1}, \cdot\right)\right\|_{L^{\frac{2 n}{n+2}}\left(M_{0}\right)} \mathrm{d} y_{1} .
\end{aligned}
$$

Using Lemma 2.3, we estimate

$$
\sup _{k \leq \sqrt{\lambda_{j}}<k+1}\left|m_{\tau}\left(t, \sqrt{\lambda_{j}}\right)\right| \leq \frac{1}{k} \begin{cases}\mathrm{e}^{-(k-\tau)|t|} & \text { when } \tau<k \\ 1 & \text { when } k \leq \tau<k+1 \\ \mathrm{e}^{-(\tau-k-1)|t|} & \text { when } \tau \geq k+1\end{cases}
$$


with $k>0$. (Note that when $k=0$, a majorant is $\mathrm{e}^{-(\tau / 2)|t|}$ for $\tau \geq 4$ ). This allows us to estimate the series

$$
\begin{aligned}
\sum_{k=0}^{\infty}(1 & +k)^{1-\frac{2}{n}} \sup _{k \leq \sqrt{\lambda_{j}}<k+1}\left|m_{\tau}\left(t, \sqrt{\lambda_{j}}\right)\right| \\
& \lesssim \sum_{1 \leq k \leq \tau-2} k^{-\frac{2}{n}} \mathrm{e}^{-(\tau-k-1)|t|}+\tau^{-\frac{2}{n}}+\sum_{k>\tau+1} k^{-\frac{2}{n}} \mathrm{e}^{-(k-\tau)|t|}+e^{-(\tau / 2)|t|} \\
& \lesssim \int_{0}^{\tau-2} r^{-\frac{2}{n}} \mathrm{e}^{-(\tau-r-2)|t|} \mathrm{d} r+1+\int_{\tau}^{\infty} r^{-\frac{2}{n}} \mathrm{e}^{-(r-\tau)|t|} \mathrm{d} r
\end{aligned}
$$

By an obvious change of variables we have

$$
\begin{aligned}
& \int_{0}^{\tau-2} r^{-\frac{2}{n}} \mathrm{e}^{-(\tau-r-2)|t|} \mathrm{d} r+\int_{\tau}^{\infty} r^{-\frac{2}{n}} \mathrm{e}^{-(r-\tau)|t|} \mathrm{d} r \\
& \quad \leq 2|t|^{-1+\frac{2}{n}}\left(\int_{0}^{1} r^{-\frac{2}{n}} \mathrm{~d} r+\int_{1}^{\infty} \mathrm{e}^{-s} \mathrm{~d} s\right) \lesssim|t|^{-1+\frac{2}{n}}
\end{aligned}
$$

whence

$$
\sum_{k=0}^{\infty}(1+k)^{1-\frac{2}{n}} \sup _{k \leq \sqrt{\lambda_{j}}<k+1}\left|m_{\tau}\left(t, \sqrt{\lambda_{j}}\right)\right| \lesssim 1+|t|^{-1+\frac{2}{n}} .
$$

From the estimates (2.8) and (2.9), we obtain

$$
\begin{aligned}
\| G_{\tau} & f\left(x_{1}, \cdot\right) \|_{L^{\frac{2 n}{n-2}}\left(M_{0}\right)} \\
& \lesssim \int_{-\infty}^{\infty}\left(1+\left|x_{1}-y_{1}\right|^{-1+\frac{2}{n}}\right)\left\|f\left(y_{1}, \cdot\right)\right\|_{L^{\frac{2 n}{n+2}}\left(M_{0}\right)} \mathrm{d} y_{1} \\
& \lesssim \int_{-\infty}^{\infty}\left|x_{1}-y_{1}\right|^{-1+\frac{2}{n}}\left\|f\left(y_{1}, \cdot\right)\right\|_{L^{\frac{2 n}{n+2}}\left(M_{0}\right)} \mathrm{d} y_{1}+|I|^{\frac{1}{2}-\frac{1}{n}}\|f\|_{L^{\frac{2 n}{n+2}}\left(I \times M_{0}\right)}
\end{aligned}
$$

and we conclude using the Hardy-Littlewood-Sobolev inequality

$$
\left\|G_{\tau} f\right\|_{L^{\frac{2 n}{n-2}\left(I \times M_{0}\right)}} \lesssim\|f\|_{L^{\frac{2 n}{n+2}\left(I \times M_{0}\right)}} .
$$

This completes the proof of Proposition2.1.

\section{Complex Geometrical optics}

In this section we will construct the complex geometrical optics solutions that will be used to recover an $L^{n / 2}$ potential. Throughout the section, let $(M, g)$ be a compact manifold with smooth boundary, and let $(M, g) \Subset(T, g) \Subset(\tilde{T}, g)$ where $T=\mathbf{R} \times M_{0}, \tilde{T}=\mathbf{R} \times \tilde{M}_{0}$, and $g=e \oplus g_{0}$, and $\left(M_{0}, g_{0}\right) \Subset\left(\tilde{M}_{0}, g_{0}\right)$ are two $(n-1)$-dimensional simple manifolds. We also assume that $n \geq 3$. 
First we state a consequence of Proposition 2.1 for the manifold $M$ (this follows easily by embedding $\left(\tilde{M}_{0}, g_{0}\right)$ in some compact manifold without boundary and using suitable restrictions and extensions by zero).

Proposition 3.1. For $|\tau| \geq 4$ outside a countable set, there is a linear operator $G_{\tau}: L^{2}(M) \rightarrow H^{2}(M)$ such that

$$
\begin{array}{ll}
\mathrm{e}^{\tau x_{1}}\left(-\Delta_{g}\right) \mathrm{e}^{-\tau x_{1}} G_{\tau} v=v & \text { for } v \in L^{2}(M), \\
G_{\tau} \mathrm{e}^{\tau x_{1}}\left(-\Delta_{g}\right) \mathrm{e}^{-\tau x_{1}} v=v & \text { for } v \in C_{0}^{\infty}\left(M^{\text {int }}\right) .
\end{array}
$$

This operator satisfies

$$
\begin{aligned}
\left\|G_{\tau} f\right\|_{L^{2}(M)} & \leq \frac{C_{0}}{|\tau|}\|f\|_{L^{2}(M)}, \\
\left\|G_{\tau} f\right\|_{H^{1}(M)} & \leq C_{0}\|f\|_{L^{2}(M)}, \\
\left\|G_{\tau} f\right\|_{L^{\frac{2 n}{n-2}}(M)} & \leq C_{0}\|f\|_{L^{\frac{2 n}{n+2}}(M)},
\end{aligned}
$$

where $C_{0}$ is independent of $\tau$.

Let us first construct the required complex geometrical optics solutions for the case where no potential is present. This is analogous to [4, Proposition 5.1] for $q=0$.

Proposition 3.2. Let $\omega \in \tilde{M}_{0} \backslash M_{0}$ be a fixed point, let $\lambda \in \mathbf{R}$ be fixed, and let $b \in C^{\infty}\left(S^{n-2}\right)$ be a function. Write $x=\left(x_{1}, r, \theta\right)$ where $(r, \theta)$ are polar normal coordinates with center $\omega$ in $\left(\tilde{M}_{0}, g_{0}\right)$. For $|\tau|$ sufficiently large outside a countable set, there exists $u_{0} \in H^{1}(M)$ satisfying

$$
\begin{aligned}
-\Delta_{g} u_{0} & =0 \quad \text { in } M, \\
u_{0} & =\mathrm{e}^{-\tau x_{1}}\left(\mathrm{e}^{-i \tau r}|g|^{-1 / 4} \mathrm{e}^{i \lambda\left(x_{1}+i r\right)} b(\theta)+r_{0}\right)
\end{aligned}
$$

where $r_{0}$ satisfies

$$
|\tau|\left\|r_{0}\right\|_{L^{2}(M)}+\left\|r_{0}\right\|_{H^{1}(M)}+\left\|r_{0}\right\|_{L^{\frac{2 n}{n-2}(M)}} \lesssim 1 .
$$

Proof. The claim follows if one can find $r_{0}$ satisfying

$$
\mathrm{e}^{\tau x_{1}}\left(-\Delta_{g}\right) \mathrm{e}^{-\tau x_{1}} r_{0}=f
$$

with the required norm estimates, where

$$
f=\mathrm{e}^{\tau x_{1}} \Delta_{g} \mathrm{e}^{-\tau x_{1}}\left(\mathrm{e}^{-i \tau r} \mathrm{e}^{i \lambda\left(x_{1}+i r\right)} b(\theta)\right) .
$$

It is enough to take $r_{0}=G_{\tau} f$ and to note that

$$
\|f\|_{L^{2}(M)}=\left\|\Delta_{g}\left(\mathrm{e}^{i \lambda\left(x_{1}+i r\right)} b(\theta)\right)\right\|_{L^{2}(M)} \lesssim 1
$$


The $L^{2}$ and $H^{1}$ estimates follow from Proposition 3.1. The $L^{\frac{2 n}{n-2}}$ estimate follows from the $H^{1}$ estimate and Sobolev embedding, or alternatively from the $L^{\frac{2 n}{n+2}} \rightarrow L^{\frac{2 n}{n-2}}$ estimate for $G_{\tau}$.

We next consider the case with a potential $q \in L^{n / 2}(M)$, and try to find a solution to $\left(-\Delta_{g}+q\right) u=0$ in $M$ of the form

$$
u=u_{0}+\mathrm{e}^{-\tau x_{1}} r_{1} \text {. }
$$

Since $-\Delta_{g} u_{0}=0$, the function $r_{1}$ should satisfy

$$
\mathrm{e}^{\tau x_{1}}\left(-\Delta_{g}+q\right) \mathrm{e}^{-\tau x_{1}} r_{1}=-q \mathrm{e}^{\tau x_{1}} u_{0} .
$$

Since $q$ is only in $L^{n / 2}(M)$, here we need to use the $L^{\frac{2 n}{n+2}} \rightarrow L^{\frac{2 n}{n-2}}$ estimates for $G_{\tau}$. We follow the argument of Lavine and Nachman [18]. It will be convenient to symmetrize the situation slightly. Later on, the $L^{n}$ functions $m_{j}$ in the next lemma are chosen to be essentially $|q|^{1 / 2}$.

Lemma 3.3. Let $m_{1}, m_{2} \in L^{n}(M)$ be two fixed functions. Then for $|\tau| \geq \tau_{0}$ outside a countable set,

$$
\left\|m_{1} G_{\tau} m_{2} f\right\|_{L^{2}} \leq C_{0}\left\|m_{1}\right\|_{L^{n}}\left\|m_{2}\right\|_{L^{n}}\|f\|_{L^{2}} .
$$

Further,

$$
\left\|m_{1} G_{\tau} m_{2} f\right\|_{L^{2}(M) \rightarrow L^{2}(M)} \rightarrow 0
$$

as $|\tau| \rightarrow \infty$.

Proof. The Hölder inequality and Proposition 3.1 imply that

$$
\begin{aligned}
\left\|m_{1} G_{\tau} m_{2} f\right\|_{L^{2}} & \leq\left\|m_{1}\right\|_{L^{n}}\left\|G_{\tau} m_{2} f\right\|_{L^{\frac{2 n}{n-2}}} \leq C_{0}\left\|m_{1}\right\|_{L^{n}}\left\|m_{2} f\right\|_{L^{\frac{2 n}{n+2}}} \\
& \leq C_{0}\left\|m_{1}\right\|_{L^{n}}\left\|m_{2}\right\|_{L^{n}}\|f\|_{L^{2}} .
\end{aligned}
$$

Let $\varepsilon>0$ and decompose $m_{j}=m_{j}^{\sharp}+m_{j}^{b}$ where $m_{j}^{\sharp} \in L^{\infty}(M)$,

$$
\text { and } \quad \begin{aligned}
\left\|m_{j}^{\sharp}\right\|_{L^{n}} & \leq\left\|m_{j}\right\|_{L^{n}} \\
\text { and } & \left\|m_{j}^{b}\right\|_{L^{n}} \leq \frac{\varepsilon}{3 C_{0} \max \left(\left\|m_{1}\right\|_{L^{n}},\left\|m_{2}\right\|_{L^{n}}\right)} .
\end{aligned}
$$

(One can take for instance $m_{j}^{\sharp}=m_{j} \chi_{\left\{\left|m_{j}\right| \leq \mu\right\}}$ for large enough $\mu$.) It follows from the $L^{2}$ estimates for $G_{\tau}$ and (3.2) that

$$
\begin{aligned}
\left\|m_{1} G_{\tau} m_{2} f\right\|_{L^{2}} & \leq\left\|m_{1}^{\sharp} G_{\tau} m_{2}^{\sharp} f\right\|_{L^{2}}+\left\|m_{1}^{\sharp} G_{\tau} m_{2}^{b} f\right\|_{L^{2}}+\left\|m_{1}^{b} G_{\tau} m_{2} f\right\|_{L^{2}} \\
& \leq\left(\frac{C_{0}\left\|m_{1}^{\sharp}\right\|_{L^{\infty}}\left\|m_{2}^{\sharp}\right\|_{L^{\infty}}}{|\tau|}+\frac{\varepsilon}{3}+\frac{\varepsilon}{3}\right)\|f\|_{L^{2}} .
\end{aligned}
$$

The last expression is bounded by $\varepsilon\|f\|_{L^{2}}$ if $|\tau|$ is sufficiently large. This proves (3.3). 
We now finish the construction of complex geometrical optics solutions.

Proposition 3.4. Assume that $q \in L^{n / 2}(M)$. Let $\omega \in \tilde{M}_{0} \backslash M_{0}$ be a fixed point, let $\lambda \in \mathbf{R}$ be fixed, and let $b \in C^{\infty}\left(S^{n-2}\right)$ be a function. Write $x=\left(x_{1}, r, \theta\right)$ where $(r, \theta)$ are polar normal coordinates with center $\omega$ in $\left(\tilde{M}_{0}, g_{0}\right)$. For $|\tau|$ sufficiently large outside a countable set, there exists a solution $u \in H^{1}(M)$ of $\left(-\Delta_{g}+q\right) u=0$ in $M$ of the form

$$
u=\mathrm{e}^{-\tau x_{1}}\left(\mathrm{e}^{-i \tau r}|g|^{-1 / 4} \mathrm{e}^{i \lambda\left(x_{1}+i r\right)} b(\theta)+\tilde{r}\right)
$$

where $\tilde{r}$ satisfies

$$
\|\tilde{r}\|_{L^{\frac{2 n}{n-2}(M)}} \lesssim 1, \quad\|\tilde{r}\|_{L^{2}(M)} \rightarrow 0 \text { as }|\tau| \rightarrow \infty .
$$

Proof. As explained above, we let $u_{0}$ be the harmonic function given in Proposition 3.2, and look for a solution of the form $u=u_{0}+\mathrm{e}^{-\tau x_{1}} r_{1}$. We write $q(x)=|q(x)| \mathrm{e}^{i \alpha(x)}=|q(x)|^{1 / 2} m(x)$ where $m(x)=|q(x)|^{1 / 2} \mathrm{e}^{i \alpha(x)}$. Then $|q|^{1 / 2}, m \in L^{n}(M)$ with $L^{n}$ norms equal to $\|q\|_{L^{n / 2}}^{1 / 2}$.

We obtain a solution $u$ provided that (3.1) holds. Trying $r_{1}$ in the form $r_{1}=G_{\tau}|q|^{1 / 2} v$, we see that $v$ should satisfy

$$
\left(\mathrm{Id}+m G_{\tau}|q|^{1 / 2}\right) v=-m \mathrm{e}^{\tau x_{1}} u_{0} .
$$

By Lemma 3.3, for $|\tau|$ sufficiently large one has $\left\|m G_{\tau}|q|^{1 / 2}\right\|_{L^{2} \rightarrow L^{2}} \leq$ $1 / 2$. One then obtains a solution

$$
v=-\left(\mathrm{Id}+m G_{\tau}|q|^{1 / 2}\right)^{-1}\left(m \mathrm{e}^{\tau x_{1}} u_{0}\right) .
$$

Since $\left\|m \mathrm{e}^{\tau x_{1}} u_{0}\right\|_{L^{2}} \leq\|m\|_{L^{n}}\left\|\mathrm{e}^{\tau x_{1}} u_{0}\right\|_{L^{\frac{2 n}{n-2}}} \lesssim 1$, it follows that $\|v\|_{L^{2}} \lesssim$ 1. Consequently

$$
\left\|r_{1}\right\|_{L^{\frac{2 n}{n-2}}} \leq C_{0}\left\||q|^{1 / 2} v\right\|_{L^{\frac{2 n}{n+2}}} \lesssim 1
$$

Now $u$ is of the form given in the statement of the proposition, provided that

$$
\tilde{r}=r_{0}+r_{1} .
$$

This remainder term satisfies $\|\tilde{r}\|_{L^{\frac{2 n}{n-2}}} \lesssim 1$.

To study $\|\tilde{r}\|_{L^{2}}$ we fix $\varepsilon>0$ and make a decomposition $|q|^{1 / 2}=s^{\sharp}+s^{b}$ where $s^{\sharp} \in L^{\infty}(M),\left\|s^{\sharp}\right\|_{L^{n}} \leq\|q\|_{L^{n / 2}}^{1 / 2}$, and $\left\|s^{b}\right\|_{L^{n}} \leq \varepsilon$. Then

$$
\begin{aligned}
\left\|r_{1}\right\|_{L^{2}} & \leq\left\|G_{\tau} s^{\sharp} v\right\|_{L^{2}}+C_{1}\left\|G_{\tau} s^{b} v\right\|_{L^{\frac{2 n}{n-2}}} \\
& \leq\left(\frac{C_{0}\left\|s^{\sharp}\right\|_{L^{\infty}}}{|\tau|}+C_{0} C_{1}\left\|s^{b}\right\|_{L^{n}}\right)\|v\|_{L^{2}} .
\end{aligned}
$$

Choosing $|\tau|$ sufficiently large, we see that $\left\|r_{1}\right\|_{L^{2}} \lesssim \varepsilon$ for $|\tau|$ large. Since also $\left\|r_{0}\right\|_{L^{2}(M)} \lesssim|\tau|^{-1}$, it follows that $\|\tilde{r}\|_{L^{2}} \rightarrow 0$ as $|\tau| \rightarrow \infty$. 
Finally, to prove that $u \in H^{1}(M)$, it is enough to consider a compact manifold $(\hat{M}, g)$ which is slightly larger than $(M, g)$ and extend $q$ by zero outside $M$, and to perform the above construction of solutions in $\hat{M}$. One obtains a solution $u \in L^{\frac{2 n}{n-2}}(\hat{M}) \subseteq L^{2}(\hat{M})$, and $\Delta_{g} u=q u \in$ $L^{\frac{2 n}{n+2}}(\hat{M}) \subseteq H^{-1}(\hat{M})$ by Sobolev embedding. Elliptic regularity implies that $u \in H_{\text {loc }}^{1}\left(\hat{M}^{\text {int }}\right)$, thus also $u \in H^{1}(M)$.

\section{ReCOVERING THE POTENTIAL}

We are now ready to give the proof of the main uniqueness result.

Proof of Theorem 1.1. Assume, as before, that $(M, g) \Subset(T, g) \Subset(\tilde{T}, g)$ where $T=\mathbf{R} \times M_{0}, \tilde{T}=\mathbf{R} \times \tilde{M}_{0}$, and $\left(M_{0}, g_{0}\right) \Subset\left(\tilde{M}_{0}, g_{0}\right)$ are two $(n-1)$-dimensional simple manifolds where $n \geq 3$. Also assume that $g=e \oplus g_{0}$.

From the assumption $\Lambda_{g, q_{1}}=\Lambda_{g, q_{2}}$, writing $q=q_{1}-q_{2}$, we know from Lemma ?? that

$$
\int_{M} q u_{1} u_{2} \mathrm{~d} V_{g}=0
$$

where $u_{1}, u_{2} \in H^{1}(M)$ are solutions of $\left(-\Delta_{g}+q_{1}\right) u_{1}=0$ in $M$ and $\left(-\Delta_{g}+q_{2}\right) u_{2}=0$ in $M$. By Proposition 3.4, for $\tau$ sufficiently large outside a countable set there exist solutions $u_{j}$ of the form

$$
\begin{aligned}
& u_{1}=\mathrm{e}^{-\tau\left(x_{1}+i r\right)}\left(|g|^{-1 / 4} \mathrm{e}^{i \lambda\left(x_{1}+i r\right)} b(\theta)+r_{1}\right), \\
& u_{2}=\mathrm{e}^{\tau\left(x_{1}+i r\right)}\left(|g|^{-1 / 4}+r_{2}\right) .
\end{aligned}
$$

Here $\lambda$ is a fixed real number, $b \in C^{\infty}\left(S^{n-2}\right)$ is a fixed function, and $x=\left(x_{1}, r, \theta\right)$ are coordinates in $\tilde{T}$ where $(r, \theta)$ are polar normal coordinates in $\left(\tilde{M}_{0}, g_{0}\right)$ with center at a fixed point $\omega \in \tilde{M}_{0} \backslash M_{0}$. Also, the remainder terms satisfy

$$
\left\|r_{j}\right\|_{L^{\frac{2 n}{n-2}(M)}}=O(1), \quad\left\|r_{j}\right\|_{L^{2}(M)}=o(1)
$$

as $\tau \rightarrow \infty$.

Inserting the solutions in (4.1) and noting that $\mathrm{d} V_{g}=|g|^{1 / 2} \mathrm{~d} x_{1} \mathrm{~d} r \mathrm{~d} \theta$, we obtain that

$$
\int_{M} q \mathrm{e}^{i \lambda\left(x_{1}+i r\right)} b(\theta) \mathrm{d} x_{1} \mathrm{~d} r \mathrm{~d} \theta=\int_{M} q\left(a_{1} r_{2}+a_{2} r_{1}+r_{1} r_{2}\right) \mathrm{d} V
$$

where $a_{1}, a_{2}$ are smooth functions in $M$ independent of $\tau$. We show that the right hand side converges to 0 as $\tau \rightarrow \infty$. To do this, fix 
$\varepsilon>0$ and write $q=q^{\sharp}+q^{b}$ where $q^{\sharp} \in L^{\infty}(M),\left\|q^{\sharp}\right\|_{L^{n / 2}} \leq\|q\|_{L^{n / 2}}$, and $\left\|q^{b}\right\|_{L^{n / 2}} \leq \varepsilon$. The right hand side of (4.2) is bounded by

$$
\begin{aligned}
\mid \int_{M} q\left(a_{1} r_{2}\right. & \left.+a_{2} r_{1}+r_{1} r_{2}\right) \mathrm{d} V \mid \\
& \lesssim\left\|q^{\sharp}\right\|_{L^{\infty}}\left(\left\|r_{1}\right\|_{L^{2}}+\left\|r_{2}\right\|_{L^{2}}+\left\|r_{1}\right\|_{L^{2}}\left\|r_{2}\right\|_{L^{2}}\right) \\
& +\left\|q^{b}\right\|_{L^{n / 2}}\left(\left\|r_{1}\right\|_{L^{\frac{2 n}{n-2}}}+\left\|r_{2}\right\|_{L^{\frac{2 n}{n-2}}}+\left\|r_{1}\right\|_{L^{\frac{2 n}{n-2}}}\left\|r_{2}\right\|_{L^{\frac{2 n}{n-2}}}\right) .
\end{aligned}
$$

Using the bounds for $r_{j}$, if $\tau$ is sufficiently large then the last quantity is $\lesssim \varepsilon$. This shows that the right hand side of (4.2) goes to 0 as $\tau \rightarrow \infty$.

Extend $q$ by zero into $T$ and interpret the left hand side of (4.2) as an integral over $T$. Taking the limit as $\tau \rightarrow \infty$, we obtain that

$$
\int_{-\infty}^{\infty} \int_{0}^{\infty} \int_{S^{n-2}} q\left(x_{1}, r, \theta\right) \mathrm{e}^{i \lambda\left(x_{1}+i r\right)} b(\theta) \mathrm{d} x_{1} \mathrm{~d} r \mathrm{~d} \theta=0 .
$$

This statement is true for all choices of $\omega \in \tilde{M}_{0} \backslash M_{0}$, for all real numbers $\lambda$, and for all functions $b \in C^{\infty}\left(S^{n-2}\right)$. Since $q \in L^{1}(M)$, Fubini's theorem shows that $q(\cdot, r, \theta)$ is in $L^{1}(\mathbf{R})$ for a.e. $(r, \theta)$. Consequently

$$
\int_{S^{n-2}} \int_{0}^{\infty} f_{\lambda}(r, \theta) \mathrm{e}^{-\lambda r} b(\theta) \mathrm{d} r \mathrm{~d} \theta=0
$$

where $f_{\lambda} \in L^{1}\left(M_{0}\right)$ is the function given by

$$
f_{\lambda}(r, \theta)=\int_{-\infty}^{\infty} \mathrm{e}^{i \lambda x_{1}} q\left(x_{1}, r, \theta\right) \mathrm{d} x_{1}
$$

If $|\lambda|$ is sufficiently small, it follows from Lemma 5.1 below that the vanishing of the integrals (4.3) for all choices $\omega$ and $b$ implies that $f_{\lambda}=0$. Since $q(\cdot, r, \theta)$ is a compactly supported function in $L^{1}(\mathbf{R})$ for a.e. $(r, \theta)$, the Paley-Wiener theorem shows that $q(\cdot, r, \theta)=0$ for such $(r, \theta)$. Consequently $q_{1}=q_{2}$.

\section{Attenuated Ray transform}

It remains to show the following lemma which was used in the proof of Theorem 1.1.

Lemma 5.1. Let $\left(M_{0}, g_{0}\right)$ be an $(n-1)$-dimensional simple manifold, and let $f \in L^{1}\left(M_{0}\right)$. Consider the integrals

$$
\int_{S^{n-2}} \int_{0}^{\tau(\omega, \theta)} f(r, \theta) \mathrm{e}^{-\lambda r} b(\theta) \mathrm{d} r \mathrm{~d} \theta
$$

where $(r, \theta)$ are polar normal coordinates in $\left(M_{0}, g_{0}\right)$ centered at some $\omega \in \partial M_{0}$, and $\tau(\omega, \theta)$ is the time when the geodesic $r \mapsto(r, \theta)$ exits $M_{0}$. 
If $|\lambda|$ is sufficiently small, and if these integrals vanish for all $\omega \in \partial M_{0}$ and all $b \in C^{\infty}\left(S^{n-2}\right)$, then $f=0$.

The last result is related to the vanishing of the attenuated geodesic ray transform of the function $f$ on $M_{0}$. For the following facts see [3], [21], [23]. To define the ray transform, we consider the unit sphere bundle

$$
S M_{0}=\bigcup_{x \in M_{0}} S_{x}, \quad S_{x}=\left\{(x, \xi) \in T_{x} M_{0} ;|\xi|=1\right\} .
$$

This manifold has boundary $\partial\left(S M_{0}\right)=\left\{(x, \xi) \in S M_{0} ; x \in \partial M_{0}\right\}$ which is the union of the sets of inward and outward pointing vectors,

$$
\partial_{ \pm}\left(S M_{0}\right)=\left\{(x, \xi) \in S M_{0} ; \pm\langle\xi, \nu\rangle \leq 0\right\} .
$$

Here $\nu$ is the outer unit normal vector to $\partial M_{0}$. Note that $\partial_{+}\left(S M_{0}\right)$ is a manifold whose boundary consists of all the tangential directions $\left\{(x, \xi) \in \partial\left(S M_{0}\right) ;\langle\xi, \nu\rangle=0\right\}$. Thus the space $C_{0}^{\infty}\left(\left(\partial_{+}\left(S M_{0}\right)\right)^{\mathrm{int}}\right)$ contains all smooth functions on $\partial_{+}\left(S M_{0}\right)$ vanishing near tangential directions.

Denote by $t \mapsto \gamma(t, x, \xi)$ the unit speed geodesic starting at $x$ in direction $\xi$, and let $\tau(x, \xi)$ be the time when this geodesic exits $M_{0}$. Since $\left(M_{0}, g_{0}\right)$ is simple, $\tau(x, \xi)$ is finite for each $(x, \xi) \in S M_{0}$. We also write $\varphi_{t}(x, \xi)=(\gamma(t, x, \xi), \dot{\gamma}(t, x, \xi))$ for the geodesic flow.

The geodesic ray transform, with constant attenuation $-\lambda$, acts on functions on $M_{0}$ by

$$
T_{\lambda} f(x, \xi)=\int_{0}^{\tau(x, \xi)} f(\gamma(t, x, \xi)) \mathrm{e}^{-\lambda t} \mathrm{~d} t, \quad(x, \xi) \in \partial_{+}\left(S M_{0}\right) .
$$

In Lemma 5.1, if $f$ were a continuous function, one could choose $b(\theta)$ to approximate a delta function at fixed angles $\theta$ and obtain that

$$
\int_{0}^{\tau(\omega, \theta)} f(r, \theta) \mathrm{e}^{-\lambda r} \mathrm{~d} r=0
$$

for any $\omega \in \partial M_{0}$ and any $\theta \in S^{n-2}$. Since $(r, \theta)$ are polar normal coordinates the curves $r \mapsto(r, \theta)$ are geodesics in $\left(M_{0}, g_{0}\right)$, and this would imply that

$$
T_{\lambda} f(x, \xi)=0 \quad \text { for all }(x, \xi) \in \partial_{+}\left(S M_{0}\right) .
$$

One has the following injectivity result from [4, Theorem 7.1]. (If $M_{0}$ is two-dimensional the smallness assumption on the attenuation coefficient was recently removed in [22].) 
Proposition 5.2. Let $\left(M_{0}, g_{0}\right)$ be a simple manifold. There exists $\varepsilon>0$ such that if $\lambda$ is a real number with $|\lambda|<\varepsilon$ and if $f \in C^{\infty}(M)$, then the condition $T_{\lambda} f(x, \xi)=0$ for all $(x, \xi) \in \partial_{+}\left(S M_{0}\right)$ implies that $f=0$.

The previous argument together with Proposition 5.2 proves Lemma 5.1 for smooth $f$. However, this requires well defined restrictions of $f$ to all geodesics and it is not obvious how to do this when $f \in L^{1}$. We circumvent this problem by using duality and the ellipticity of the normal operator $T_{\lambda}^{*} T_{\lambda}$.

We will need a few facts. Below we write

$$
h_{\psi}(x, \xi)=h\left(\varphi_{-\tau(x,-\xi)}(x, \xi)\right), \quad(x, \xi) \in S M_{0}
$$

for $h \in C^{\infty}\left(\partial_{+}\left(S M_{0}\right)\right)$, and

$$
(h, \tilde{h})_{L_{\mu}^{2}\left(\partial_{+}\left(S M_{0}\right)\right)}=\int_{\partial_{+}\left(S M_{0}\right)} h \tilde{h} \mu \mathrm{d}\left(\partial\left(S M_{0}\right)\right)
$$

where $\mu(x, \xi)=-\langle\xi, \nu(x)\rangle$ and $\mathrm{d} N$ is the natural Riemannian volume form on a manifold $N$.

Lemma 5.3. (Santaló formula) If $F: S M_{0} \rightarrow \mathbf{R}$ is continuous then

$$
\begin{aligned}
& \int_{S M_{0}} F \mathrm{~d}\left(S M_{0}\right) \\
& \quad=\int_{\partial_{+}\left(S M_{0}\right)} \int_{0}^{\tau(x, \xi)} F\left(\varphi_{t}(x, \xi)\right) \mu(x, \xi) \mathrm{d} t \mathrm{~d}\left(\partial\left(S M_{0}\right)\right)(x, \xi) .
\end{aligned}
$$

Proof. See [3, Lemma A.8].

Lemma 5.4. If $f \in C^{\infty}\left(M_{0}\right)$ and $h \in C_{0}^{\infty}\left(\left(\partial_{+}\left(S M_{0}\right)\right)^{\text {int }}\right)$ then

$$
\left(T_{\lambda} f, h\right)_{L_{\mu}^{2}\left(\partial_{+}\left(S M_{0}\right)\right)}=\left(f, T_{\lambda}^{*} h\right)_{L^{2}\left(M_{0}\right)}
$$

where

$$
T_{\lambda}^{*} h(x)=\int_{S_{x}} \mathrm{e}^{-\lambda \tau(x,-\xi)} h_{\psi}(x, \xi) \mathrm{d} S_{x}(\xi), \quad x \in M_{0}
$$


Proof. By the Santaló formula

$$
\begin{aligned}
\left(T_{\lambda} f, h\right)_{L_{\mu}^{2}\left(\partial_{+}\left(S M_{0}\right)\right)} & \int_{\partial_{+}\left(S M_{0}\right)} \int_{0}^{\tau(x, \xi)} \mathrm{e}^{-\lambda t} f(\gamma(t, x, \xi)) h \mu \mathrm{d} t \mathrm{~d}\left(\partial\left(S M_{0}\right)\right) \\
& =\int_{\partial_{+}\left(S M_{0}\right)} \int_{0}^{\tau(x, \xi)} \mathrm{e}^{-\lambda t} f\left(\varphi_{t}(x, \xi)\right) h_{\psi}\left(\varphi_{t}(x, \xi)\right) \mu \mathrm{d} t \mathrm{~d}\left(\partial\left(S M_{0}\right)\right) \\
& =\int_{S M_{0}} \mathrm{e}^{-\lambda \tau(x,-\xi)} f(x) h_{\psi}(x, \xi) \mathrm{d}\left(S M_{0}\right) \\
& =\int_{M_{0}} f(x)\left(\int_{S_{x}} \mathrm{e}^{-\lambda \tau(x,-\xi)} h_{\psi}(x, \xi) \mathrm{d} S_{x}(\xi)\right) \mathrm{d} V(x) .
\end{aligned}
$$

This proves Lemma 5.4.

Lemma 5.5. $T_{\lambda}^{*} T_{\lambda}$ is a self-adjoint elliptic pseudodifferential operator of order -1 in $M_{0}^{\text {int }}$.

Proof. This is contained in [6, Proposition 2].

Proof of Lemma 5.1. The first step is to extend $\left(M_{0}, g_{0}\right)$ to a slightly larger simple manifold and to extend $f$ by zero. In this way we can assume that $f$ is compactly supported in $M_{0}^{\text {int }}$.

We let $b$ also depend on $\omega$ and change notations to write the assumption in the lemma in the form

$$
\int_{S_{x}} \int_{0}^{\tau(x, \xi)} \mathrm{e}^{-\lambda t} f(\gamma(t, x, \xi)) b(x, \xi) \mathrm{d} t \mathrm{~d} S_{x}(\xi)=0
$$

for all $x \in \partial M_{0}$ and $b \in C_{0}^{\infty}\left(\left(\partial_{+}\left(S M_{0}\right)\right)^{\text {int }}\right)$. Next we make the choice $b(x, \xi)=h(x, \xi) \mu(x, \xi)$ for $h \in C_{0}^{\infty}\left(\left(\partial_{+}\left(S M_{0}\right)\right)^{\text {int }}\right)$ and integrate the last identity over $\partial M_{0}$ to obtain

$$
\int_{\partial_{+}\left(S M_{0}\right)} \int_{0}^{\tau(x, \xi)} \mathrm{e}^{-\lambda t} f(\gamma(t, x, \xi)) h(x, \xi) \mu \mathrm{d} t \mathrm{~d}\left(\partial\left(S M_{0}\right)\right)=0 .
$$

We are now in the same situation as in the proof of Lemma 5.4, and invoking the Santaló formula implies

$$
\int_{M_{0}} f(x) T_{\lambda}^{*} h(x) \mathrm{d} V(x)=0
$$

for all $h \in C_{0}^{\infty}\left(\left(\partial_{+}\left(S M_{0}\right)\right)^{\text {int }}\right)$. Note that the last integral is absolutely convergent because $f \in L^{1}\left(M_{0}\right)$, and also the previous steps are justified by Fubini's theorem. 
It remains to choose $h=T_{\lambda} \varphi$ for $\varphi \in C_{0}^{\infty}\left(M_{0}^{\text {int }}\right)$ to obtain that

$$
\int_{M_{0}} f(x) T_{\lambda}^{*} T_{\lambda} \varphi(x) \mathrm{d} V(x)=0 .
$$

Since $T_{\lambda}^{*} T_{\lambda}$ is self-adjoint, we have

$$
\int_{M_{0}}\left(T_{\lambda}^{*} T_{\lambda} f(x)\right) \varphi(x) \mathrm{d} V(x)=0 .
$$

This is valid for all test functions $\varphi$, so $T_{\lambda}^{*} T_{\lambda} f=0$. By ellipticity, since $f$ was compactly supported in $M_{0}^{\text {int }}$, it follows that $f \in C_{0}^{\infty}\left(M_{0}^{\text {int }}\right)$. One can now use the argument for smooth $f$ given above, together with the injectivity result (Proposition 5.2), to conclude the proof that $f=0$.

\section{REFERENCES}

[1] A. P. Calderón, On an inverse boundary value problem, Seminar on Numerical Analysis and its Applications to Continuum Physics, Soc. Brasileira de Matemática, Río de Janeiro, 1980.

[2] S. Chanillo, A problem in electrical prospection and a n-dimensional BorgLevinson theorem, Proc. Amer. Math. Soc. 108 (1990), 761-767.

[3] N. S. Dairbekov, G. P. Paternain, P. Stefanov, and G. Uhlmann, The boundary rigidity problem in the presence of a magnetic field, Adv. Math. 216 (2007), 535-609.

[4] D. Dos Santos Ferreira, C. E. Kenig, M. Salo, and G. Uhlmann, Limiting Carleman weights and anisotropic inverse problems, Invent. Math. 178 (2009), 119-171.

[5] D. Dos Santos Ferreira, C. E. Kenig, J. Sjöstrand, and G. Uhlmann, Determining a magnetic Schrödinger operator from partial Cauchy data, Comm. Math. Phys. 271 (2007), 467-488.

[6] B. Frigyik, P. Stefanov, and G. Uhlmann, The X-ray transform for a generic family of curves and weights, J. Geom. Anal. 18 (2008), 89-108.

[7] C. Guillarmou, A. Sa Barreto, Inverse problems for Einstein manifolds, Inverse Probl. Imaging 3 (2009), 1-15.

[8] D. Gilbarg, N. S. Trudinger, Elliptic partial differential equations of second order, Springer-Verlag, 2001.

[9] L. Hörmander, The analysis of linear partial differential operators, Springer Verlag, 1994.

[10] D. Jerison, Carleman inequalities for the Dirac and Laplace operators and unique continuation, Adv. Math. 63 (1986), 118-134.

[11] D. Jerison and C. E. Kenig, Unique continuation and absence of positive eigenvalues for Schrödinger operators, Ann. of Math. 121 (1985), 463-494.

[12] A. Katchalov, Y. Kurylev, M. Lassas, Inverse boundary spectral problems, Monographs and Surveys in Pure and Applied Mathematics 123, Chapman Hall/CRC-press, 2001.

[13] C. E. Kenig, A. Ruiz, and C. D. Sogge, Uniform Sobolev inequalities and unique continuation for second order constant coefficient differential operators, Duke Math. J. 55 (1987), 329-347. 
[14] C. E. Kenig, M. Salo, and G. Uhlmann, Inverse problems for the anisotropic Maxwell equations,Duke Math. J. 157 (2011), 369-419.

[15] C. E. Kenig, J. Sjöstrand, and G. Uhlmann, The Calderón problem with partial data, Ann. of Math. 165 (2007), 567-591.

[16] H. Koch and D. Tataru, Carleman estimates and unique continuation for second order elliptic equations with nonsmooth coefficients, Comm. Pure Appl. Math. 54 (2001), no. 3, 339-360.

[17] H. Koch and D. Tataru, Dispersive estimates for principally normal pseudodifferential operators, Comm. Pure Appl. Math. 58 (2005), 217-284.

[18] R. Lavine and A. Nachman, unpublished (personal communication), announced in A. I. Nachman, Inverse scattering at fixed energy, Proceedings of the Xth Congress on Mathematical Physics, L. Schmdgen (Ed.), Leipzig, Germany, 1991, 434-441, Springer-Verlag.

[19] J. Lee, G. Uhlmann, Determining anisotropic real-analytic conductivities by boundary measurements, Comm. Pure Appl. Math., 42 (1989), 1097-1112.

[20] A. Nachman, Reconstructions from boundary measurements, Ann. of Math. 128 (1988), 531-576.

[21] L. Pestov, G. Uhlmann, Two dimensional compact simple Riemannian manifolds are boundary distance rigid, Ann. of Math. 161 (2005), 1089-1106.

[22] M. Salo, G. Uhlmann, The attenuated ray transform on simple surfaces, J. Diff. Geom. (to appear), arXiv:1004.2323.

[23] V. A. Sharafutdinov, Integral geometry of tensor fields, in Inverse and Ill-Posed Problems Series, VSP 1994.

[24] Z. Shen, On absolute continuity of the periodic Schrödinger operators, IMRN 1 (2001), 1-31.

[25] C. D. Sogge, Concerning the $L^{p}$ norm of spectral clusters for second-order elliptic operators on compact manifolds, J. Funct. Anal. 77 (1988), 123-138.

[26] C. D. Sogge, Strong uniqueness theorems for second order elliptic differential equations, Amer. J. Math. 112 (1990), 943-984.

[27] C. D. Sogge, Fourier integrals in classical analysis, Cambridge University Press, 1993.

[28] E. M. Stein, Harmonic Analysis: real variable methods, orthogonality and oscillatory integrals, Princeton University Press, 1993.

[29] J. Sylvester, An anisotropic inverse boundary value problem, Comm. Pure Appl. Math. 43 (1990), 201-232.

[30] J. Sylvester and G. Uhlmann, A global uniqueness theorem for an inverse boundary value problem, Ann. of Math. 125 (1987), 153-169.

[31] G. Uhlmann, Electrical impedance tomography and Calderón's problem, Inverse Problems 25 (2009), 123011.

Université Paris 13, Cnrs, Umr 7539 Laga, 99, avenue Jean-Baptiste

Clément, F-93430 Villetaneuse, France

E-mail address: ddsf@math.univ-paris13.fr

Department of Mathematics, University of Chicago, 5734 UniverSity Avenue, Chicago, IL 60637-1514, USA

E-mail address: cek@math.uchicago.edu 
Department of Mathematics and Statistics, University of Helsinki, PO Box 68, 00014 Helsinki, Finland

E-mail address: mikko.salo@helsinki.fi 